\title{
Molecular dynamics simulations of the properties of water-methanol mixtures. Effects of force fields
}

\author{
M. Cruz Sanchez $z^{1}$, H. Domingue $z^{2}, \mathrm{O}^{2}$. Pizio ${ }^{1 / *}$ \\ ${ }^{1}$ Instituto de Química, Universidad Nacional Autónoma de México, Circuito Exterior, \\ 04510 Cd. de México, México \\ 2 Instituto de Investigaciones en Materiales, Universidad Nacional Autónoma de México, Circuito Exterior, \\ 04510 Cd. de México, México
}

Received February 22, 2019

\begin{abstract}
Isothermal-isobaric molecular dynamics simulations are used to examine the microscopic structure and some properties of water-methanol liquid mixture. The TIP4P/2005 and SPC/E water models are combined with the united atom TraPPE and the all-atom force field model for methanol. Our principal focus is to evaluate the quality of predictions of different combinations of model force fields concerning the composition dependence of basic properties of this system. Specifically, we explored the composition effects on density, excess molar volume and excess entropy, as well as on the surface tension and static dielectric constant. In addition, the structural properties are described in terms of the coordination numbers and the average number of hydrogen bonds between molecules of constituent species. Finally, the composition dependence of self-diffusion coefficients of the species is evaluated. All theoretical predictions are tested with respect to experimental data.
\end{abstract}

Key words: water-methanol mixtures, mixing properties, surface tension, molecular dynamics simulations

PACS: 61.20.-p, 61.20-Gy, 61.20.Ja

\section{Introduction}

This manuscript is the first part of our two-stage project that involves water-methanol mixtures. Namely, in this first part, we present a set of results coming from the isothermal-isobaric (NPT) computer simulation concerning composition changes of density, excess mixing volume and entropy, first coordination numbers of species and average number of hydrogen bonds. Moreover, we explore the behavior of the surface tension and the dielectric constant on composition, as well as the self-diffusion coefficients of species.

The forthcoming, second part of the project, is devoted to the exploration of changes of all the properties mentioned above, brought by addition of $\mathrm{NaCl}$ salt to water-methanol solvent. Again, the NPT computer simulation technique will be applied. One of the principal issues we would like to address is to evaluate the validity and quality of theoretical results coming from different combinations of force fields with respect to experimental data.

For specific purposes of our project, it is worth mentioning that molecular dynamics computer simulations have been widely applied to mixtures of water with various solvents. Most frequently studied seem to be the mixtures of water with alcohols, specifically the water-methanol mixtures, see, e.g., [111] and references therein for a rather comprehensive account of the previously applied modelling. Our present report has been inspired by most recent contributions concerning the system in question [6, 8, 9]. On the other hand, important experimental observations concerning this system and used in the present study have been discussed in [12]-17].

\footnotetext{
*Corresponding author, E-mail: oapizio@gmail.com
} 
The majority of computer simulation studies cited above have been focused on the application of a single combination of force fields describing each constituent species, water and methanol with the exception of [9]. This latter work involved two methanol force fields, namely the TraPPE model [18] and OPLS-all atom model [19]. As concerns water, the SPC/E and TIP4P models were chosen, see, e.g., [20] and [21], respectively. Formally, we apply similar strategy. However, in contrast to [9], we apply the TIP4P/2005 water model [22] rather than the TIP4P version [21]. It is known that the the TIP4P/2005 version provides a better performance of the microscopic structure of water [23] and yields even better description of various properties compared to the TIP4P, see, e.g., table 2 of reference [24]. Moreover, we are specifically interested in using the TIP4P/2005, because it has been quite recently applied to parametrize the description of properties of $\mathrm{NaCl}$ aqueous solution, see [25, 26]. It opens up the possibility to study $\mathrm{NaCl}$ solutions with water-methanol solvent in subsequent work. On the other hand, the gained knowledge would permit to explore complex solutes in mixed solvents tuning with confidence their solubility on solvent composition. Some specific molecules of interest in medicinal chemistry, for example curcumin, are marginally soluble in water, but dissolve well in, e.g., alcohols or dimethylsulfoxide [27-29]. Additional comments concerning the methodological difference of our procedure and technical details in comparison with reference [9] are given in the body of the manuscript below.

To summarize, the principal objective of the present report is to investigate a set of properties of watermethanol mixtures in the entire interval of composition. All the properties are validated by comparison with experimental results.

\section{Models and simulation details}

Preliminarily discussing the simulation methodology, for the sake of convenience for the reader, in table 1 we list some of the models used in previous studies of water-methanol mixtures and related to the present work. Within the united atom model for methanol, like OPLS/UA and TraPPE (see table 1), the $\mathrm{CH}_{3}$ group is considered as a single site. On the other hand, the all atom models explicitly involve all the hydrogens of the methanol molecule.

Table 1. Models of water-methanol liquid mixtures and the combination rules (according to GROMACS nomenclature).

\begin{tabular}{|c|c|c|c|}
\hline & water & methanol & combination rules \\
\hline Galicia-Andres et al. [3] & $\begin{array}{l}\text { TIP4P/Ew [30] } \\
\text { SPC/E [20] }\end{array}$ & OPLS/UA [31, 32] & CR2 (L-B) \\
\hline Galicia-Andres et al.. [2] & $\begin{array}{l}\text { TIP4P/Ew [30] } \\
\text { SPC/E [20] }\end{array}$ & OPLS/AA [19] & CR3 \\
\hline Wensink et al. [1] & TIP4P [21] & OPLS/AA [19] & CR3 \\
\hline Guevara-Carrion et al. [33] & $\begin{array}{l}\text { SPC/E [20] } \\
\text { TIP4P/2005 [22] }\end{array}$ & UA-own design [34] & CR2 (L-B) \\
\hline Kohns et al.. [35] & SPC/E [20] & UA-own design [34] & CR2 (L-B) \\
\hline Požar et al. [36] & SPC/E [20] & TraPPE [18] & CR2 (L-B) \\
\hline Obeidat et al. [9] & $\begin{array}{l}\text { TIP4P [21] } \\
\text { SPC/E [20] }\end{array}$ & $\begin{array}{l}\text { TraPPE [18] } \\
\text { OPLS/AA [19] }\end{array}$ & CR2 (L-B) \\
\hline
\end{tabular}

\subsection{Technical details}

In this work, we explore the SPC/E model [20] and the TIP4P/2005 model [22] for water. For methanol, we used two models, namely the united atom model-TraPPE [18], and all atom model [19], denominated as MET/TraPPE, and MET/AA, respectively. The Lorentz-Berthelot combination rules are used to determine the cross parameters. 
The long-range electrostatic interactions were handled by the particle mesh Ewald method implemented in the GROMACS software package (fourth order, Fourier spacing equal to 0.12) with a precision of $10^{-5}$. The nonbonded interactions were cut off at $1.4 \mathrm{~nm}$. The van der Waals tail correction terms to the energy and pressure were taken into account. In order to maintain the geometry of the water and methanol molecules, the LINCS algorithm was used.

Our calculations were performed in the isothermal-isobaric $(N P T)$ ensemble at 1 bar, and at a temperature of 298.15 K. We used the GROMACS software package [37], version 5.1.2. Concerning the procedure, a periodic cubic simulation box was set up for each system. The GROMACS genbox tool was employed to randomly place all particles in the simulation box. To remove the possible overlaps of particles introduced by the procedure of preparation of the initial configuration, each system underwent energy minimization using the steepest descent algorithm implemented in the GROMACS package. Minimization was followed by a $50 \mathrm{ps} N P T$ equilibration run at $298.15 \mathrm{~K}$ and 1 bar using a timepstep of $0.25 \mathrm{fs}$. We used the Berendsen thermostat and barostat with $\tau_{T}=1 \mathrm{ps}$ and $\tau_{P}=1 \mathrm{ps}$ during equilibration. Constant value of $4.5 \cdot 10^{-5} \mathrm{bar}^{-1}$ for the compressibility of the mixtures was employed. In the case of pure methanol solvent, the compressibility was taken to be $1.2 \cdot 10^{-4} \mathrm{bar}^{-1}$. The V-rescale thermostat and Parrinello-Rahman barostat with $\tau_{T}=0.5 \mathrm{ps}$ and $\tau_{P}=2.0 \mathrm{ps}$ and the time step 2 fs were used during production runs. Statistics for each mole solvent composition and various ions concentration for any of the properties were collected over several $10 \mathrm{~ns} N P T$ runs, each starting from the last configuration of the preceding run. The time extension for each series of calculations will be mentioned below in the appropriate place but not less than $70 \mathrm{~ns}$.

While exploring the composition changes, the total number of molecules was kept fixed at 3000. The composition is described by the mole fraction of methanol molecules $X_{\mathrm{m}}, X_{\mathrm{m}}=N_{\mathrm{m}} /\left(N_{\mathrm{m}}+N_{\mathrm{w}}\right)$.

\section{Results and Discussion}

In order to make things clear from the very beginning, we study four models of water-methanol liquid mixtures described by the following force fields: TIP4P/2005-MET/TraPPE, TIP4P/2005-MET/AA, SPC/E-MET/TraPPE and SPC/E-MET/AA. In all cases, the Lorentz-Berthelot combination rules are used to obtain cross interaction parameters. The behavior of mixture density on composition, $X_{\mathrm{m}}$, is given in figure 1]. Our NPT computer simulation results are supplemented by the experimental data [14, 38]. It can be seen that the density is perfectly well described by the TIP4P/2005-MET/TraPPE model. The SPC/E-MET/TraPPE combination of force fields yields only a bit worse predictions in the interval of intermediate compositions. Two models, the TIP4P/2005-MET/AA and SPC/E-MET/AA, are less accurate regarding the density dependence on composition, apparently because the density of pure liquid methanol is not described accurately within the MET/AA model. These trends of behavior have been mentioned recently in [2, 3] exploring TIP4P/Ew water model [30] combined with methanol models with a different degree of sophistication. On the other hand, the density dependence on composition from the NPT simulations by Soetens and Bopp also agrees with the experimental data perfectly well [8]. It is worth mentioning that the authors used well established $\mathrm{BJH}$ [39] and $\mathrm{PHH}$ [40] flexible models for water and methanol, respectively. The results presented in figures 2 and 3 of [9] are slightly off all the observations mentioned above concerning the density dependence on methanol molar fractions. Namely, in figure 2 of that work, the liquid density of pure methanol from TraPPE and OPLS-AA models at $300 \mathrm{~K}$ looks too low compared to the values reported in TraPPE database and by other authors. Moreover, the discrepancy between the simulation predictions and experimental data given in figure 3 of [9] (the $y$-scale of this figure seems to be not very appropriate to appreciate the total density) is too big compared to other works. Apparently, trends of behavior of density from this work [9] are affected by the number of particles, system size, method of calculations using a rather small liquid slab surrounded by vacuum and by simulation time. To summarize the discussion of our figure 1 and the observations of other authors, it seems that the dependence of density on composition is one of the important properties, but not too demanding computationally, if the system size and time of simulations are properly chosen.

Next, in the spirit of previous works from our laboratory [2, 3] and of the development by Soetens and Bopp [8], we turn our attention to the mixing properties. The excess molar volume, $\Delta V_{\text {mix }}$, is defined 


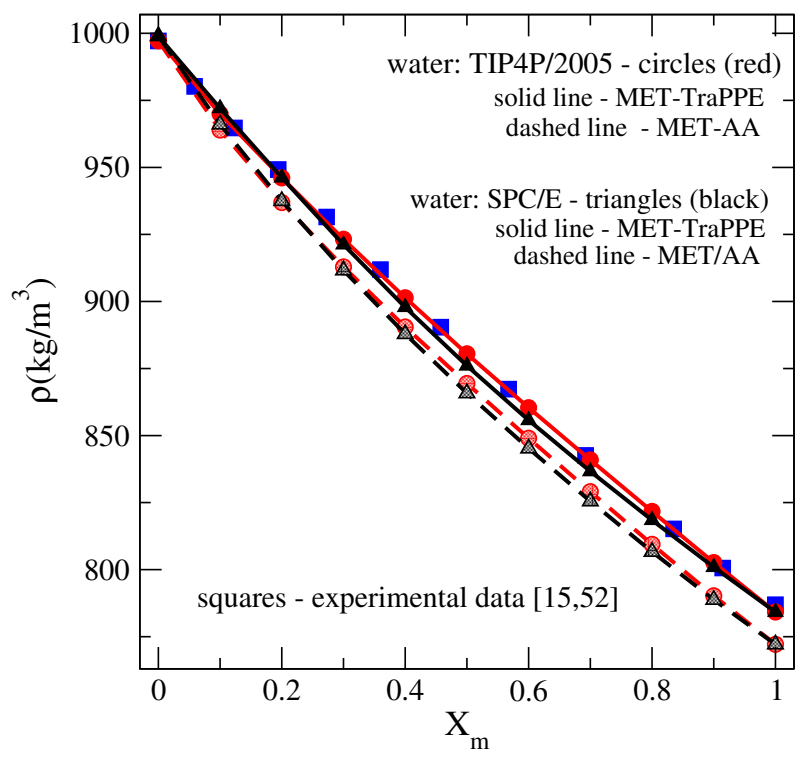

Figure 1. (Colour online) Composition dependence of water-methanol mixture density from the present NPT simulations of the TIP4P/2005-MET/TraPPE, TIP4P/2005-MET/AA, SPC/E-MET/TraPPE, and SPC/E-MET/AA models, together with experimental data [14, 38]. The nomenclature of lines and symbols is given in the figure.

as,

$$
\Delta V_{\mathrm{mix}}=V_{\mathrm{mix}}-\left(1-X_{\mathrm{m}}\right) V_{\mathrm{w}}-X_{\mathrm{m}} V_{\mathrm{m}}
$$

where $V_{\text {mix }}$ is the volume of the mixture at a certain composition, $V_{\mathrm{w}}$ and $V_{\mathrm{m}}$ refer to the molar volumes of pure water and pure methanol, respectively. Our simulation results for $\Delta V_{\text {mix }}$ are shown in figure 2(a). As in the case of density, cf. figure 1 , here we observe again that the TIP4P/2005-MET/TraPPE model yields the best agreement with experimental results [16]. The entire set of experimental points is well reproduced, slight overestimation of the magnitude of $\Delta V_{\mathrm{mix}}$ is observed in the interval of intermediate compositions and when methanol content is higher than of water. Nevertheless, the experimentally observed minimum at $X_{\mathrm{m}}=0.5$ is reproduced by simulations. If methanol is described by all-atom model (MET/AA), the agreement between simulation results and experimental points deteriorates, the minimum shifts to $X_{\mathrm{m}} \approx 0.6$. Quite similar dependence of $\Delta V_{\mathrm{mix}}$ on composition (to the TIP4P/2005-MET/AA force field) has been obtained from simulations of flexible BJH-PHH model, cf. figure 2 of reference [8]. If one combines the SPC/E water with each of two methanol models in question, the values for $\Delta V_{\text {mix }}$ in almost entire range of composition are underestimated, figure 2(a). This kind of inaccuracy has been observed previously and was discussed in detail in references [3, 41]. Energetic aspects of mixing are given by the excess enthalpy of mixing, $\Delta H_{\mathrm{mix}}$. It is defined similarly to equation 1 .

$$
\Delta H_{\text {mix }}=H_{\text {mix }}-\left(1-X_{\mathrm{m}}\right) H_{\mathrm{w}}-X_{\mathrm{m}} X_{\mathrm{m}},
$$

where $H_{\text {mix }}$ refers to the mixture entalphy whereas $H_{\mathrm{w}}$ and $H_{\mathrm{m}}$ describe the entalphy of each species in pure state at the same temperature and pressure. From the comparison of the simulation results in figure 2 (b) with experimental data [16], we conclude that none of the employed force field combinations reproduce the experimental trends perfectly well. Concerning the magnitude of $\Delta H_{\mathrm{mix}}$, the TIP4P/2005MET/TraPPE and SPC/E-MET/AA models provide a better description than the TIP4P/2005-MET/AA and SPC/E-MET/TraPPE. However, all the models of this study predict the minimum of $\Delta H_{\text {mix }}$ at $X_{\mathrm{m}}$ in the interval between 0.5 and 0.6 rather than the experiment that shows the corresponding minimum at $X_{\mathrm{m}}=0.3$. Similar kind of inaccuracy has been observed and discussed for a set of previously studied models [2, 3]. Unfortunately, the excess entalphy of mixing from NPT simulations has not been presented in reference [8] for the flexible BJH-PHH model to provide a critical evaluation of the present modelling. Three data points previously reported for the excess potential energy from the NVE simulations of the 

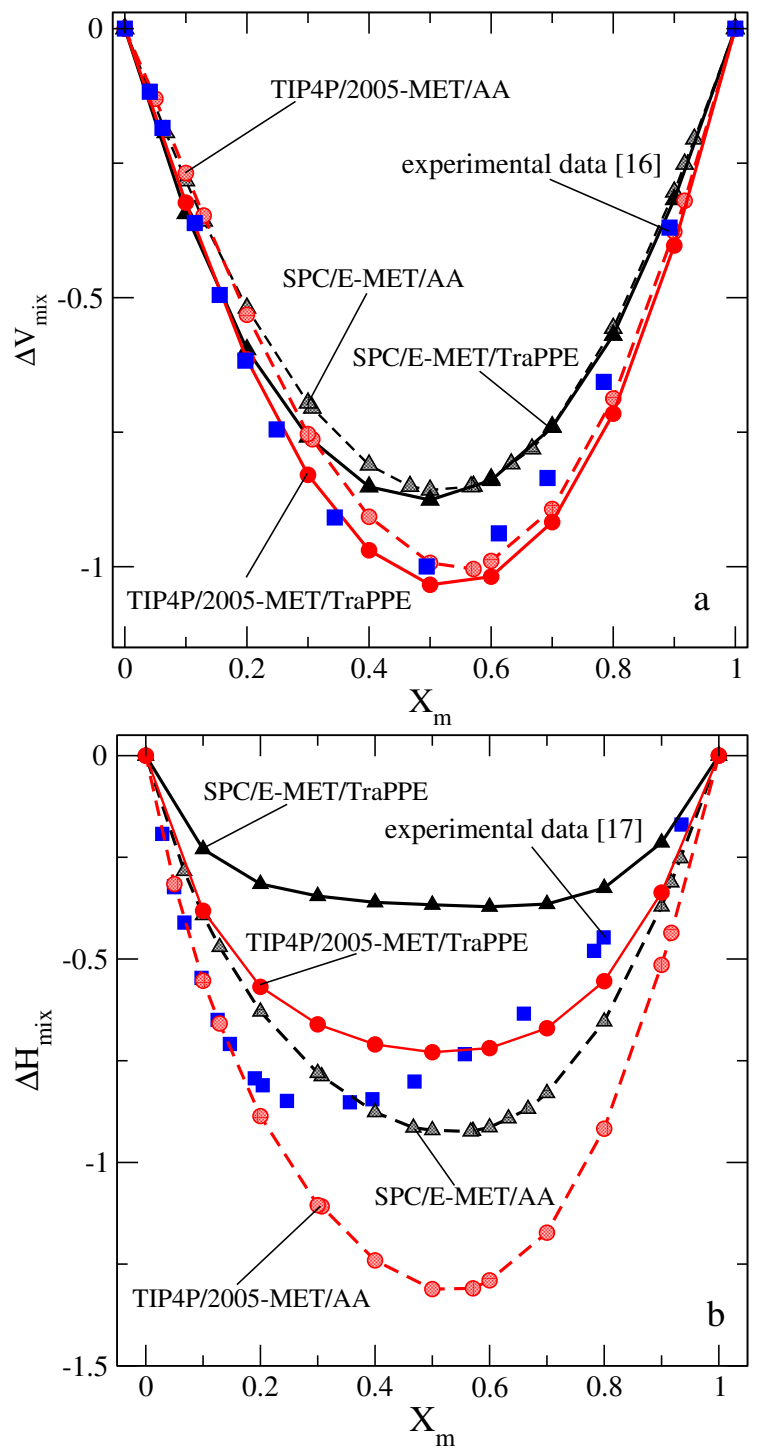

Figure 2. (Colour online) Panel (a): Excess mixing volume of water-methanol mixtures on methanol molar fraction for different combinations of models for each species together with the experimental data from reference [15] (blue squares). Panel (b): A comparison of computer simulation data for mixing entalphy with experimental data from [16] (blue squares). The nomenclature of lines and symbols is like in figure 1

combination of flexible models, see table 1 of reference [42], do not provide a definite answer in this respect, unfortunately.

The microscopic structure of water-methanol mixtures has been discussed in terms of evolution of the pair distribution functions on composition in many occasions. However, quantitative insights about the changes of structure on $X_{\mathrm{m}}$ are usually described in terms of the first coordination numbers of the species and of the number of hydrogen bonds. Here, in order to avoid unnecessary repetition, we adopt a similar point of view. The first coordination numbers of the species are defined from the running coordination numbers as common,

$$
n_{i j}(r)=4 \pi \rho_{j} \int_{0}^{r} g_{i j}(R) R^{2} \mathrm{~d} R,
$$

where the upper limit of integration in this equation is taken to correspond to the first minimum $r_{\min }$ of 
the pair distribution function $g_{i j}(r)$ of the species $i$ and $j$. A detailed description of the behavior of this property, for a set of models related to the present study, was given in [2, 3, 43]. Very recent reports also concern the evolution of the first coordination numbers, see, e.g., figure 7 of reference [8] and figures 24 and 25 of reference [9].

In order to evaluate the average number of $\mathrm{H}$-bonds between molecules, the corresponding utility of GROMACS software is applied with default distance - angle criterion. However, for distance cutoff we used the first minimum of the corresponding pair distribution function, see also discussion of this issue in references [2, 3, 43]. Averaging is performed over a piece of or the entire simulated trajectory.

Changes of $n_{i j}=n_{i j}\left(r_{\mathrm{min}}\right)$ on $X_{\mathrm{m}}$ for oxygens that belong to water or methanol, OW-OW and OW$\mathrm{OM}$, and resulting from our simulations, are shown in figure 3 (a). The coordination number $n_{\mathrm{OW}-\mathrm{OW}}$
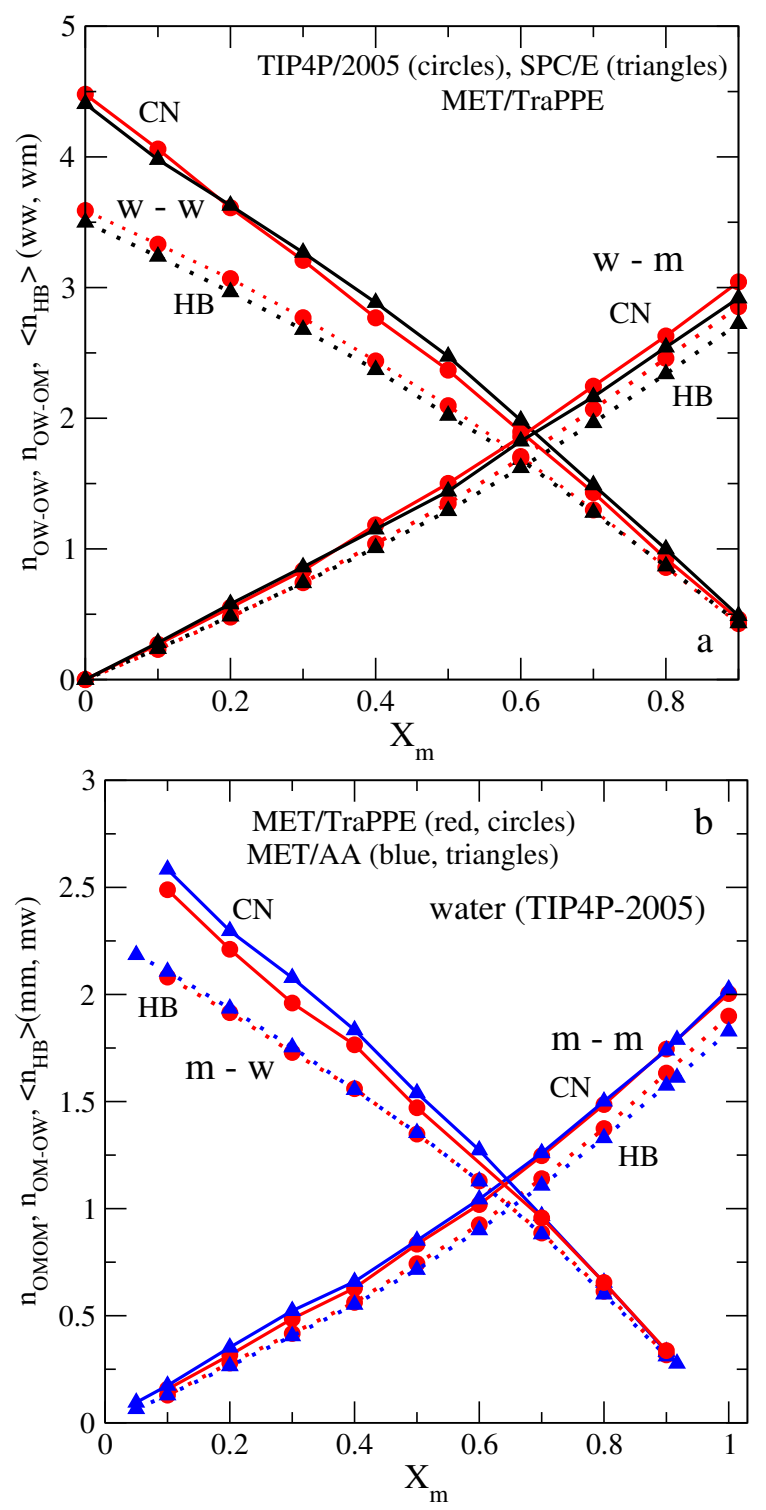

Figure 3. (Colour online) The dependence of the first coordination number of oxygens for each species $(\mathrm{CN})$ and of average number of $\mathrm{H}$-bonds $(\mathrm{HB})$ on composition for models as in figure 1 and figure 2 The coordination numbers are given by solid lines and symbols, whereas the dotted lines refer to the numbers of hydrogen bonds. Normalization of the average number of $\mathrm{H}$-bonds is performed per number of molecules of the first species of the notation: w-w, w-m and $\mathrm{m}-\mathrm{m}$ and $\mathrm{m}-\mathrm{w}$. Panel (a) is for $\mathrm{w}-\mathrm{w}$ and $\mathrm{w}-\mathrm{m}$ where the panel (b) os for $\mathrm{m}-\mathrm{m}$ and $\mathrm{m}-\mathrm{w}$. The nomenclature of lines and symbols is given in the figure. 
monotonously, almost linearly, decreases with an increasing methanol mole fraction starting from the value $\approx 4.5$ for pure water. On the other hand, $n_{\mathrm{OW}-\mathrm{OM}}$ increases up to $\approx 3$ while approaching $X_{\mathrm{m}}=1$. Two combinations of force fields, TIP4P-2005-MET/TraPPE and SPC/E-MET/TraPPE, yield almost the same results. The crossing point between $n_{\mathrm{OW}-\mathrm{OW}}\left(X_{\mathrm{m}}\right)$ and $n_{\mathrm{OW}-\mathrm{OM}}\left(X_{\mathrm{m}}\right)$ describing "inversion" in the type of predominant neighbors around water molecule occurs at $X_{\mathrm{m}} \approx 0.65$. The crossing point at the same composition characterizes the BJH-PHH combination of flexible models for two species, figure 7 of [8]. On the other hand, evolution of the average number of hydrogen bonds $\left\langle n_{\mathrm{HB}}\right\rangle$ per water molecule on the methanol mole fraction from our simulations is given in figure 3 (a) as well. Actually the dependences of $\left\langle n_{\mathrm{HB}}\right\rangle$ on $X_{\mathrm{m}}$ follow the trends of behavior of the first coordination numbers. The crossing point describing the predominant number of $\mathrm{H}$-bonds between two kinds of molecular species is observed at $X_{\mathrm{m}} \approx 0.65$. Again, two water models (TIP4P/2005 and SPC/E), if combined with MET/TraPPE, yield very similar predictions concerning the trends of behavior of $\left\langle n_{\mathrm{HB}}\right\rangle$ on $X_{\mathrm{m}}$. The "inversion" or crossover point in $n_{\text {OW-OW }}\left(X_{\mathrm{m}}\right)$ and $n_{\mathrm{OW}-\mathrm{OM}}\left(X_{\mathrm{m}}\right)$ or $\left\langle n_{\mathrm{HB}}\right\rangle$ is located close to the minimum of the excess mixing volume, cf. figure 2 (a), as it was discussed in [3]. Finally, we would like to mention that the approximation of average hydrogen bond numbers by the corresponding coordination numbers is not very appropriate in the case OW-OW, see figure 3(a), in contrast to what was claimed in [9].

Concerning the behavior of methanol coordination number and the average number of hydrogen bonds in a mixture with varying composition, figure 3 (b), we would like to mention the following trends. We explored two methanol models, the MET/TraPPE and MET/AA, combined with TIP4P2005 water model. The models yield a similar behavior. In pure methanol, the coordination number is around three and the average number of hydrogen bonds between methanol molecules is only slightly less. The methanol coordination smoothly decreases with a decreasing $X_{\mathrm{m}}$. On the other hand, the cross coordination number OM-OW increases with a decreasing $X_{\mathrm{m}}$, the corresponding hydrogen bonds number follows this behavior. The "inversion" of the composition of the surrounding of a methanol molecule on average occurs at $X_{\mathrm{m}} \approx 0.65$. In the region of low methanol mole fractions, methanol molecule incorporates the hydrogen bonded structure of water and on average forms a bit more than two hydrogen bonds with water molecules.

An excellent detailed analysis of the coordination numbers and hydrogen bonds network topology was performed in reference [44], though the energetic definition of hydrogen bonds was used in that work. Nevertheless, all trends of behavior deduced from geometric criterion for hydrogen bonding qualitatively agree with the predictions described by using energetic definition.

One of the properties representing a quite demanding test of the employed force field is the surface tension that describes the changes of surface free energy upon changing the surface area. Therefore, we have undertaken additional calculations focused on the evaluation of surface tension for a set of models under study. Relevant experimental results are available [17]. Moreover, this property has been explored in detail in several computer simulation studies of water-methanol system, see, e.g., [9. 11].

The simulations aiming at surface tension calculations at each point of composition axis in terms of $X_{\mathrm{m}}$, have been performed by using the final configuration of particles in the box from the NPT run. Next, the box edge along $z$-axis was elongated by a factor of 3 , generating a box with liquid slab and two liquid-mixture - vacuum interfaces in the $x-y$ plane, in close similarity to the procedure used in reference [45]. The total number of particles, $3 \times 10^{3}$, seem to be reasonable as it provides the area of the $x-y$ face of the liquid slab large enough to avoid size effects. The elongation of the liquid slab along $z$-axis is sufficient as well. To be more specific, we would like just to mention that the box dimensions were $4.63 \times 4.63 \times 13.89 \mathrm{~nm}^{3}$ for $X_{\mathrm{m}}=0.1$ up to $5.75 \times 5.75 \times 17.25 \mathrm{~nm}^{3}$ for $X_{\mathrm{m}}=0.9$, for the TIP4P/2005-MET/TraPPE model calculations. These numbers favourably compare with the cut-off distance for non-bonded interactions $1.4 \mathrm{~nm}$. The executable molecular dynamics file was modified by deleting fixed pressure condition, just the $V$-rescale thermostatting with the same parameters as in the $N P T$ runs has been preserved. Other corrections have not been used.

The values for the surface tension, $\gamma$, follow from the combination of the time averages for the components of the pressure tensor,

$$
\gamma=L_{z}\left\langle\left[P_{z z}-\frac{1}{2}\left(P_{x x}+P_{y y}\right)\right]\right\rangle / 2
$$

where $P_{i j}(i, j=x, y, z)$ are the components of the pressure tensor, and $\langle\ldots\rangle$ denotes the time average. 
We performed a set of $N V T$ runs, not less than 5-6, each with the time duration of $10 \mathrm{ns,}$, and obtained the results for $\gamma$ making the block average.

Our results for the surface tension and the excess mixing surface tension are given in two panels of figure 4. It is known that the TIP4P/2005 model leads to a better prediction for the surface tension of pure water in comparison with SPC/E [24]. This is also documented in figure 4 (a). Our result for pure methanol within MET/TraPPE model agrees perfectly well with reference [46]. On the other hand, the application of the same procedure with MET/AA model yields a slightly higher value for pure methanol, very close to the experimental result, figure 4(a). Small discrepancy of our result with the value given in the supplementary material to [45] can be attributed to slightly different technical details of simulations, e.g., cut-off, corrections to the electrostatics for a slab system, etc.

Overall trends of behavior of $\gamma\left(X_{\mathrm{m}}\right)$ in the entire composition interval are qualitatively correctly reproduced by all four combinations of the force fields. A better description at low values of $X_{\mathrm{m}}$ is reached if the TIP4P/2005 model is invloved. For high values of $X_{\mathrm{m}}$, the application of the MET/AA model leads to a better agreement with the experimental data. The values calculated by us are lower
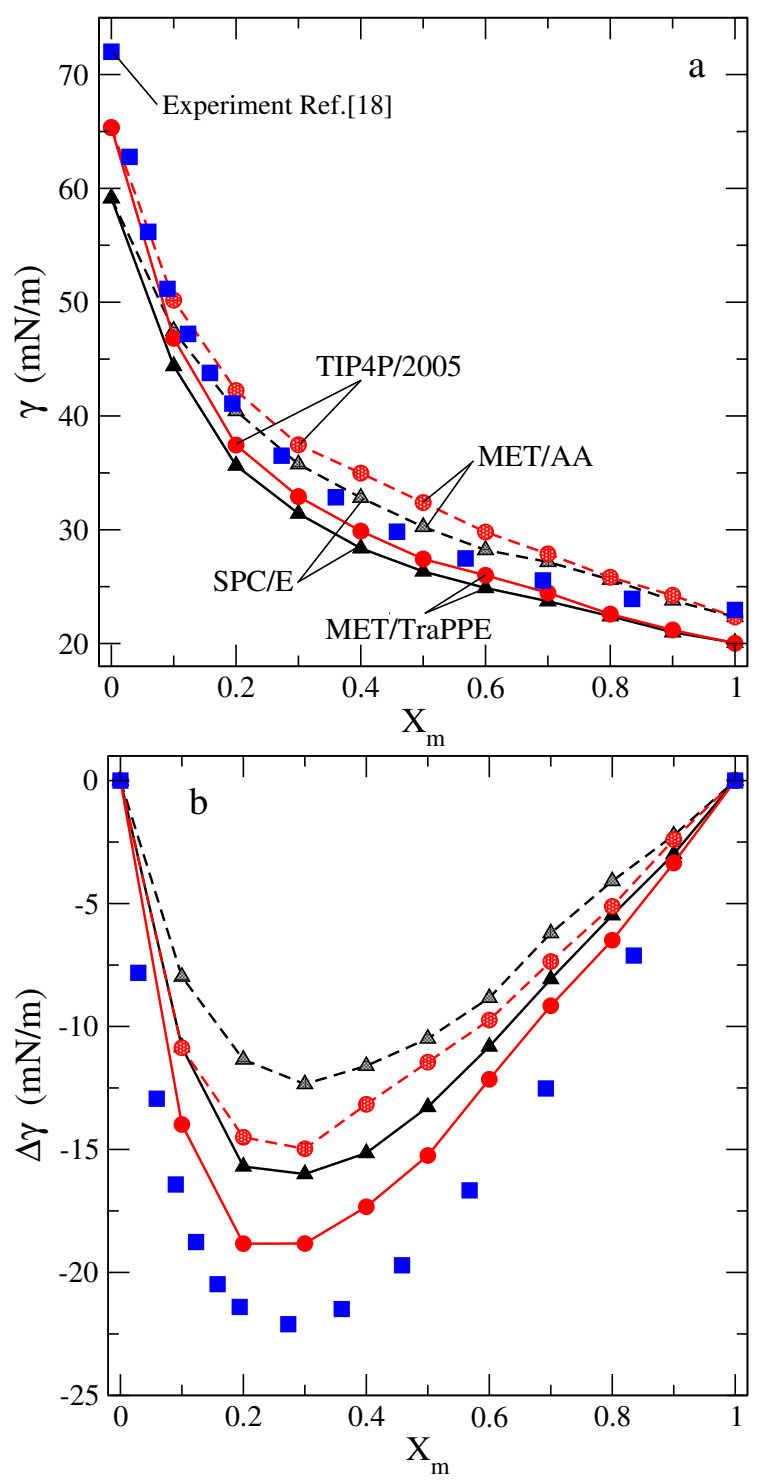

Figure 4. (Colour online) Panel (a): Surface tension of water-methanol mixtures on methanol molar fraction as compared with experimental data from reference [17] (blue squares). Panel (b): Excess mixing surface tension on composition. Both panels refer to the same models as in previous figures. 
than the experimental data [17] at all $X_{\mathrm{m}}$, due to the defficiency of performance of models for each component, if the TIP4P/2005 and MET/TraPPE are used. With the MET/AA model of methanol, the surface tension values are higher than the experimental points at intermediate and high methanol mole fractions. Interestingly, the surface tension substantially decreases even if a small amount of methanol is added to water. This behavior can be attributed to the tendency for methanol to be located close to the interface with vacuum. Our findings are in agreement with the results reported in [9] for the same system but with a smaller number of particles.

If one focuses on the deviation of the surface tension from ideal mixing behavior, figure 4 (b), the TIP4P/2005-MET/TraPPE model is the closest to the experimental predictions. The present satisfactory modelling can be seemingly used with confidence to other more complex systems. It is worth mentioning that experimental results predict a maximum absolute value of deviation from ideality at $X_{\mathrm{m}} \approx 0.3$ and computer simulation results reproduce this behavior. This particular composition actually coincides with the maximum deviation of mixing entalphy, cf. figure 2 (b), that has not been predicted by the models in question. Seemingly, the local composition fluctuations with specific orientations of molecules (yielding nonideality of surface tension) are missing in the bulk phase to provide a correct behavior of nonideality of entalphy. At present, it is difficult to offer a recipe how to improve the performance of nonpolarizable rigid models for surface tension and for nonideality of entalphy, though.

In order to explore how the particles of different species are distributed in the box when $X_{\mathrm{m}}$ changes, we have plotted the number density profiles of water and methanol oxygens in the entire slab plus vacuum system in figure 5. At a low value of $X_{\mathrm{m}}$, see $X_{\mathrm{m}}=0.1$ as an example, water is uniformly distributed in the slab. Methanol species are distributed uniformly in the inner part of the slab, though we observe a high maximum for methanol oxygens distribution at each liquid slab — vacuum interface. At intermediate composition, $X_{\mathrm{m}}=0.5$, both species are almost uniformly distributed inside the liquid slab, but the maxima of $\mathrm{O}_{\mathrm{m}}$ observed for $X_{\mathrm{m}}=0.1$ disappear, in expence of the growing amount of methanol oxygens in the part of each interface exposed to vacuum. The two profiles describing the intermediate composition, $X_{\mathrm{m}}=0.5$, are not perfectly symmetric with respect to the box center. This situation occurs very rarely but can have consequences, if one pretends to obtain the precise density of species from this kind of procedure. Specifically, in reference [9] the authors failed to estimate the liquid density both for methanol and water at $X_{\mathrm{m}}=0.3$. Finally, at $X_{\mathrm{m}}=0.9$ in figure 5, it can be seen that the interface predominantly contains methanol molecules whereas water molecules "hide" in the inner part of the liquid slab. Only a small fraction of water molecules penetrates the interfacial region.

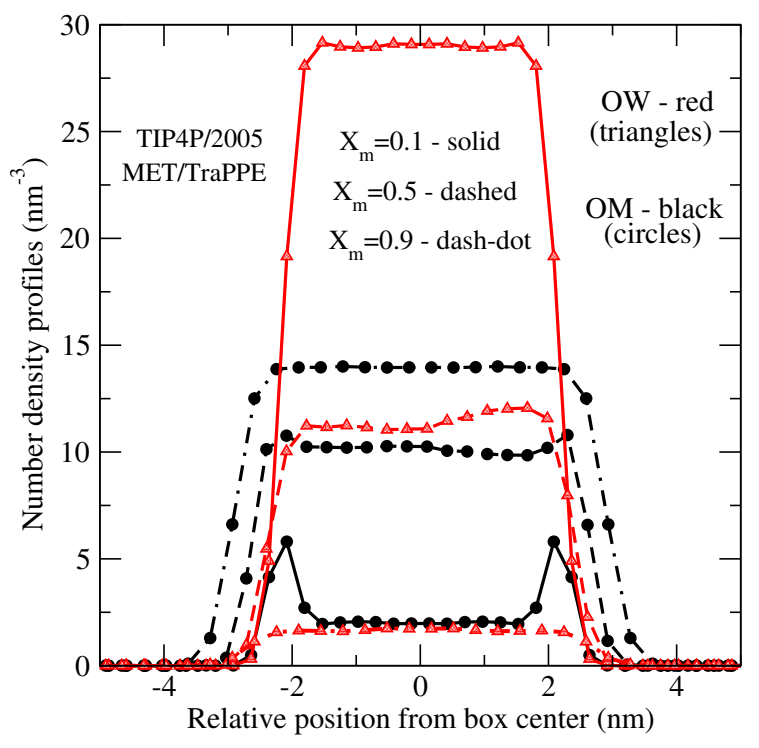

Figure 5. (Colour online) Examples of the number density profiles of water and methanol oxygens for mixtures (TIP4P/2005-MET/TraPPE model) at different composition. 
The final part of the manuscript is concerned with the description of the self-diffusion coefficients of species and changes of the dielectric constant with composition of water-methanol mixtures. The self-diffusion coefficients of water and methanol were calculated from the mean-square displacement (MSD) of a particle via Einstein relation,

$$
D_{i}=\frac{1}{6} \lim _{t \rightarrow \infty} \frac{\mathrm{d}}{\mathrm{d} t}\left\langle\left|\mathbf{r}_{i}(\tau+t)-\mathbf{r}_{i}(\tau)\right|^{2}\right\rangle
$$

where $i$ refers to water or methanol and $\tau$ denotes the time origin. Default settings of GROMACS were used for the separation of the time origins. Moreover, the fitting interval (from $10 \%$ to $50 \%$ of the analyzed trajectory) has been used to calculate $D_{\mathrm{m}}$ and $D_{\mathrm{w}}$. Moreover, a special care has been taken to fitting for the cases with a small number of particles on the extremes along the $X_{\mathrm{m}}$ axis. A set of our results is given in figure 6 .

The results for $D_{\mathrm{w}}$, are qualitatively correct, the TIP4P/2005 model slightly underestimates $D_{\mathrm{w}}$, it yields 2.1 for pure water, whereas the SPC/E overestimates $D_{\mathrm{w}}$, it yields $\approx 2.6$, see figure 6(a). Concerning the self-diffusion coefficient of pure methanol, it can be seen that MET/TraPPE underestimates $D_{\mathrm{m}}$, it
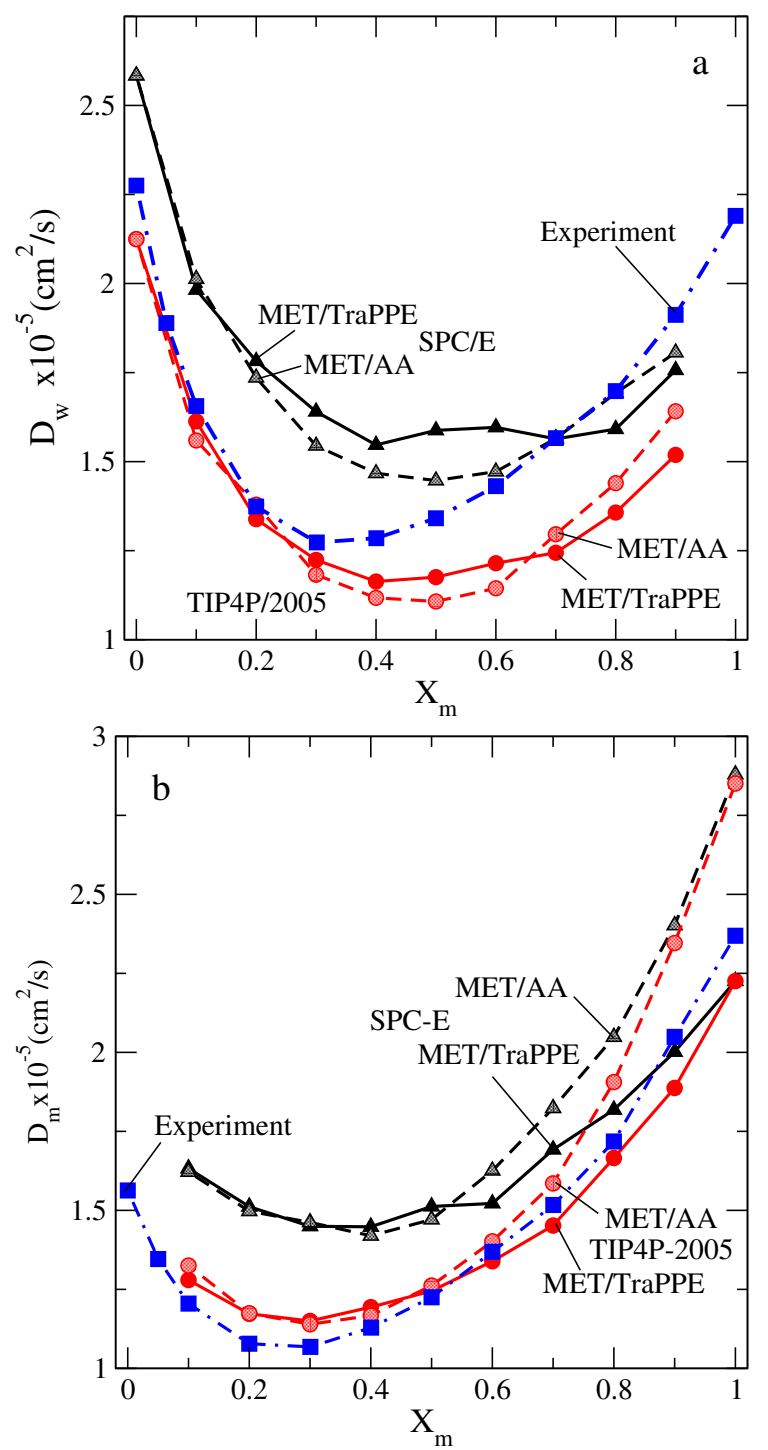

Figure 6. (Color online) Self-diffusion coefficients of water, $\mathrm{D}_{\mathrm{w}}$, and of methanol, $\mathrm{D}_{\mathrm{m}}$, in water-methanol mixture on composition [panels (a) and (b), respectively] and experimental data [47]. The nomenclature of lines and symbols is given in the figure. 
gives $\approx 2.25$, whereas the all atom model, MET/AA, substantially overestimates $D_{\mathrm{m}}$, figure 6 (b). This tendency has been already discussed in reference [43]. These inaccuracies prohibit quantitatively correct predictions for the composition changes of the self-diffusion coefficients of species.

Namely, the dependence $D_{\mathrm{w}}\left(X_{\mathrm{m}}\right)$ is qualitatively correct. The TIP4P/2005, if combined with MET/TraPPE or with MET/AA methanol model, leads to a very good prediction of $D_{\mathrm{w}}$ up to $X_{\mathrm{m}} \approx 0.3$, for higher values of $X_{\mathrm{m}}$ simulation data deviate from experimental results [47]. The miminum value for $D_{\mathrm{w}}$ from simulations is in the interval $X_{\mathrm{m}}$ between 0.4 and 0.5 whereas the experiment predicts this minimum at 0.3 . The minimum value of $D_{\mathrm{w}}$ from simulations along the composition axis coincides with the minimum of excess mixing volume at $X_{\mathrm{m}}=0.5$. At high values of $X_{\mathrm{m}}$, the growth of $D_{\mathrm{w}}$ is similar in simulations and in experiment [47]. If water is descibed in the framework of the SPC/E model, the resuts for $D_{\mathrm{w}}\left(X_{\mathrm{m}}\right)$ substantially overestimate this self-diffusion coefficient in a wide range of composition. Solely at high values of $X_{\mathrm{m}}$, the agreement with experimental values becomes more acceptable. It is worth mentioning that we were unable to get a better shape of $D_{\mathrm{w}}\left(X_{\mathrm{m}}\right)$ within the SPC/E-MET/TraPPE model. Apparently, there are two minima, one at $X_{\mathrm{m}}=0.4$ and anoter at $X_{\mathrm{m}}=0.7$. It is difficult to establish (without additional exploration of various properties) if this behavior is related to the clustering of species at local scale as it has been discussed in the experimental study [12]. Here, we would like just to mention that similar evolution of $D_{\mathrm{w}}$ on $X_{\mathrm{m}}$ was reported in our recent work [3] in the study of SPC/E model combined by OPLS/UA model of methanol [31].

Concerning the trends of behavior of the self-diffusion of methanol species in mixtures of different composition $D_{\mathrm{m}}\left(X_{\mathrm{m}}\right)$, we would like to emphasize the following. The best combination of force fields is provided by the TIP4P/2005-MET/TraPPE model. It describes the changes of the function $D_{\mathrm{m}}\left(X_{\mathrm{m}}\right)$ pretty well in the entire composition range, figure 6 (b). The minimum value of $D_{\mathrm{m}}$ is described at a correct place, $X_{\mathrm{m}} \approx 0.3$. All other combinations of the force fields exhibit defficiencies either due to the water model like SPC/E or due to all-atom modelling of methanol at MET/AA level. An overall most satisfactory picture of the dependence of the self-diffusion coefficients of two species, therefore, results if the TIP4P/2005-MET/TraPPE model is used. We believe that alternative calculations of the self-diffusion coefficients by applying velocity autocorrelation functions should lead to similar conclusions.

Our final concern is the evolution of the dielectric constant with composition. The long-range, asymptotic behavior of correlations between molecules possessing a permanent dipole moment is determined by the dielectric constant, $\varepsilon$. Usually, long molecular dynamics runs are necessary to obtain reasonable values for $\varepsilon$, because it is calculated from the time-average of the fluctuations of the total dipole moment of the system [48],

$$
\varepsilon=1+\frac{4 \pi}{3 k_{\mathrm{B}} T V}\left(\left\langle\mathbf{M}^{2}\right\rangle-\langle\mathbf{M}\rangle^{2}\right),
$$

where $k_{\mathrm{B}}$ is the Boltzmann constant and $\mathrm{V}$ is the volume of the simulation box.

The lines from our NPT simulations of $\varepsilon$ are shown in figure 7 (a). An overall behaviour of $\varepsilon\left(X_{\mathrm{m}}\right)$ is that it decreases starting from a high value for pure water to a lower value corresponding to pure methanol with an increasing $X_{\mathrm{m}}$. As it follows from the comparison of the simulation results and experimental data [49], all four combinations of the force fields substantially underestimate the values for $\varepsilon$ in the entire composition range. The reason is that the dielectric constant for two constituents, water and methanol, is essentially underestimated. It is highly probable to improve the dependence of the static dielectric constant on composition by applying the model for water specifically parametrized to reproduce the dielectric constant for water [50]. Simultaneously, it would require parametrization of the force field for methanol, see, e.g., [51]. However, common experience is that parametrization of a single property leads to worse predictions for other properties. Therefore, this issue requires additional computation efforts.

On the other hand, a sensitive test is provided by comparison of the excess dielectric constant, $\Delta \varepsilon^{\mathrm{mix}}=\varepsilon_{\mathrm{m}}-\left[X_{\mathrm{m}} \varepsilon_{\mathrm{m}}+\left(1-X_{\mathrm{m}}\right) \varepsilon_{\mathrm{w}}\right]$, with the experimental predictions [49]. Experimental points indicate a negative deviation from ideality in the entire composition range, figure 6 (b). Maximal (negative) deviation from the ideal type of behaviour reported from the experimental measurements is at $X_{\mathrm{m}} \approx 0.45$. The simulations results reproduce the position of a minimum approximately; namely, the minimum is in the interval 0.15-0.4, dependent on the combination of the force fields. These trends are in accordance with observations concerning the deviations from ideality of thermodynamic properties. The magnitude of the excess static dielectric constant is overestimated if the SPC/E model for water is used and is 

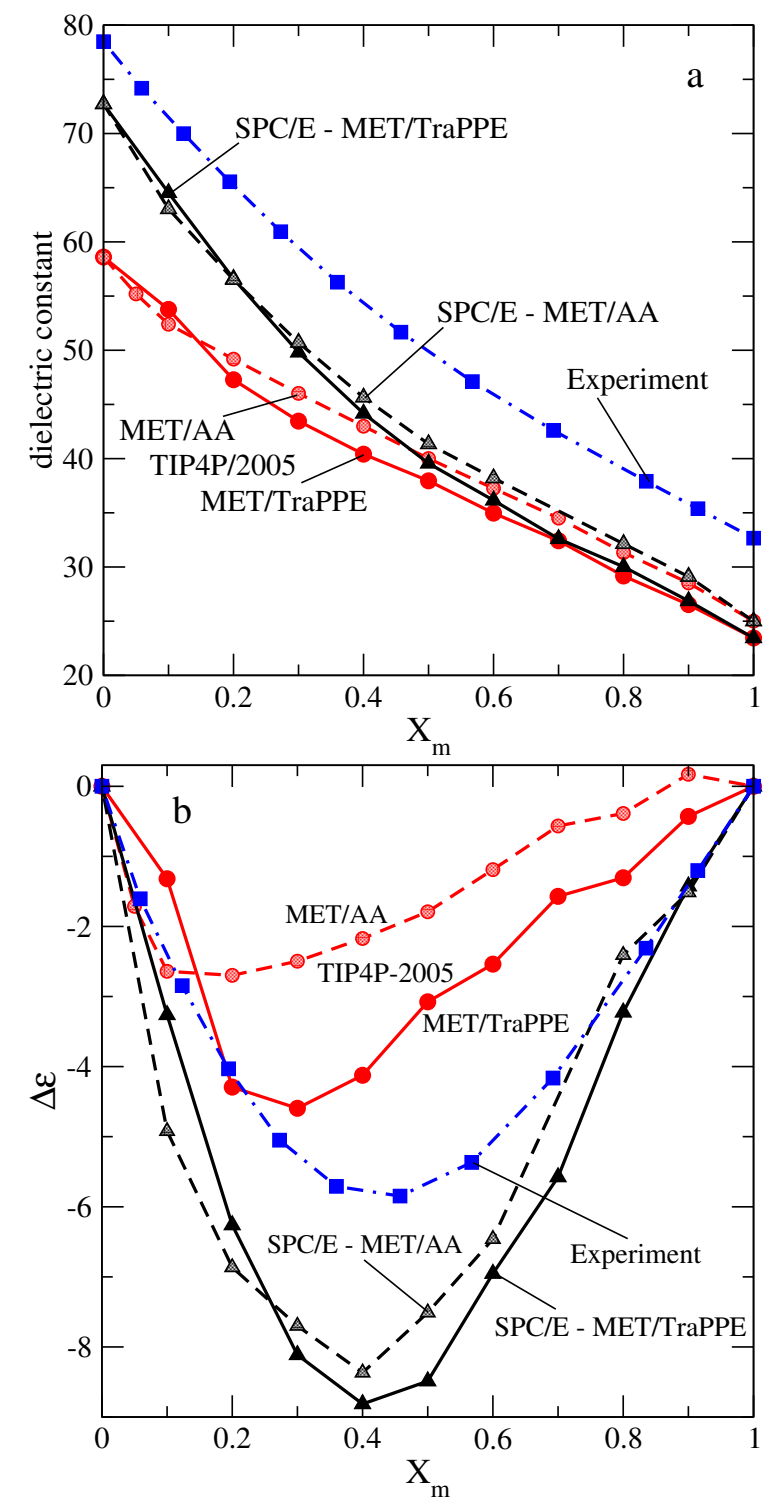

Figure 7. (Colour online) Panel (a): Dielectric constant of water-methanol mixtures on methanol molar fraction and experimental data from reference [49] (blue squares). Panel (b): Excess dielectric constant on composition. The experimental data are from [49] (blue squares). The nomenclature of lines and symbols is given in the figure.

underestimated if TIP4P/2005 is involved. The TIP4P/2005-MET/TraPPE model is the most close to the experimental data combination of models. The excess dielectric constant curve as function of chemical composition of the mixture can be related to the excess refractive index measurements, see, e.g., [52]. Consequently, a complementary comparisons with experiments would be desirable in future work.

\section{Summary and conclusions}

This work has been principally inspired by the necessity to evaluate various properties of watermethanol mixtures using a specific set of models for each component. The choice of the force fields of this study permits extensions necessary to describe ionic solutions with such combined solvents in the spirit of very recent contributions from the research laboratory of $\mathrm{C}$. Vega [25, 26] concerning aqueous $\mathrm{NaCl}$ solutions with a novel force field for ions. 
In the present work, extensive NPT molecular dynamics simulations were conducted to study thermodynamic, dynamic and structural properties of water-methanol mixtures. Two different water and two methanol nonpolarizable models were combined and simulated with the purpose of testing their predictions for an ample set of properties in the entire range of compositions. Comparisons with the available experimental data were performed. Considering the scope of the models, the predictions obtained for the mixtures appear to be qualitatively correct, particular properties were a bit better described at low methanol compositions, i.e., for water-rich compositions. However, as a general trend, it is observed that the best predictions are given with the water (TIP4P/2005)-methanol(TraPPE) mixtures since these models reasonably well predict several properties of the pure components. The results indicate that a good agreement with laboratory experiments could be obtained when both force fields, of the two components in the mixture, are good. In fact, the dielectric constant is not well predicted by any of the simulated mixtures since none of the selected models (water and methanol) predicts correctly that property.

\section{Acknowledgements}

M. Cruz and O.P. are grateful to M. Aguilar for technical support of this work at the Institute of Chemistry of the UNAM. M. Cruz acknowledges support of CONACyT of Mexico for Ph.D. scholarship.

\section{References}

1. Wensink E.J.W., Hoffmann A.C., van Maaren P.J., van der Spoel D., J. Chem. Phys., 2003, 119, 7308, doi $10.1063 / 1.1607918$

2. Galicia-Andrés E., Pusztai L., Temleitner L., Pizio O., J. Mol. Liq., 2015, 209, 586, doi $10.1016 /$ j.molliq.2015.06.045

3. Galicia-Andrés E., Dominguez H., Pusztai L., Pizio O., Condens. Matter Phys., 2015, 18, 43602, doi $10.5488 /$ CMP. 18.43602

4. Perera A., Sokolić F., Almásy L., Koga Y., J. Chem. Phys., 2006, 124, 124515, doi 10.1063/1.2178787

5. Perera A., Zoranić L., Sokolić F., Mazighi R., J. Mol. Liq., 2011, 150, 52, doi 10.1016/j.molliq.2010.05.006

6. Bakó I., Pusztai L., Temleitner L., Sci. Rep., 2017, 7, 1073, doi:10.1038/s41598-017-01095-7

7. Palinkás G., Bakó I., Heinzinger K., Bopp P., Mol. Phys., 1991, 73, 897, doi:10.1080/00268979100101641

8. Soetens J.-C., Bopp P.A., J. Phys. Chem. B, 2015, 119, 8593, doi 10.1021/acs.jpcb.5b03344

9. Obeidat A., Abu-Ghazleh H., AIP Adv., 2018, 8, 065203, doi 10.1063/1.5025575

10. Matsumoto M., Takaoka Y., Kataoka Y., J. Chem. Phys., 1993, 98, 1464, doi:10.1063/1.464310

11. Chang T.-M., Dang L.X., J. Phys. Chem. B, 2005, 109, 5759, doi $10.1021 /$ jp045649v.

12. Takamuku T., Yamaguchi T., Asato M., Matsumoto M., Nishi N., Z. Naturforsch., A: Phys. Sci., 2000, 55, 513, doi $10.1515 /$ zna-2000-0507.

13. Wakisaka A., Ohki T., Faraday Discuss., 2005, 129, 231, doi 10.1039/B405391E

14. Mikhail S.Z., Kimel W.R., J. Chem. Eng. Data, 1961, 6, 533, doi:10.1021/je60011a015.

15. McGlashan M.L., Williamson A.G., J. Chem. Eng. Data, 1976, 21, 196, doi:10.1021/je60069a019

16. Lama R.F., Lu B.C.-Y., J. Chem. Eng. Data, 1965, 10, 216, doi $10.1021 /$ je60026a003

17. Vazquez G., Alvarez E., Navaza J.M., J. Chem. Eng. Data, 1995, 40, 611, doi $10.1021 /$ je00019a016

18. Chen B., Potoff J.J., Siepmann J.I., J. Phys. Chem. B, 2001, 105, 3093, doi $10.1021 /$ jp003882x

19. Jorgensen W.L., Maxwell D.S., Tirado-Rives J., J. Am. Chem. Soc., 1996, 118, 11225, doi:10.1021/ja9621760

20. Berendsen H.J.C., Grigera J.R., Straatsma T.P., J. Phys. Chem., 1987, 91, 6269, doi $10.1021 /$ j100308a038

21. Jorgensen W.L., Chandrasekhar J., Madura J.D., Impey R.W., Klein M.L., J. Chem. Phys., 1983, 79, 926, doi $10.1063 / 1.445869$

22. Abascal J.L.F., Vega C., J. Chem. Phys., 2005, 123, 234505, doi 10.1063/1.2121687

23. Pusztai L., Pizio O., Sokolowski S., J. Chem. Phys., 2008, 129, 184103, doi:10.1063/1.2976578

24. Vega C., Abascal J.L.F., Phys. Chem. Chem. Phys., 2011, 13, 19663, doi 10.1039/C1CP22168J

25. Benavides A.L., Aragones J.L., Vega C., J. Chem. Phys., 2016, 144, 124504, doi 10.1063/1.4943780

26. Benavides A.L., Portillo M.A., Chamorro V.C., Espinosa J.R., Abascal J.L.F., Vega C., J. Chem. Phys., 2017, 147, 104501, doi $10.1063 / 1.5001190$

27. Hazra M.K., Roy S., Bagchi B., J. Chem. Phys., 2014, 141, 18C501, doi 10.1063/1.4895539

28. Samanta S., Roccatano D., J. Phys. Chem. B, 2013, 117, 3250, doi:10.1021/jp309476u

29. Patsahan T., Ilnytskyi J.M., Pizio O., Condens. Matter Phys., 2017, 20, 23003, doi:10.5488/CMP.20.23003

30. Horn H.W., Swope W.C., Pitera J.W., Madura J.D., Dick T.J., Hura G.L., Head-Gordon T., J. Chem. Phys., 2004, 120, 9665 , doi $10.1063 / 1.1683075$ 
31. Jorgensen W.L., J. Phys. Chem., 1986, 90, 1276, doi 10.1021/j100398a015.

32. Haughney M., Ferrario M., McDonald I.R., J. Phys. Chem., 1987, 91, 4934, doi 10.1021/j100303a011

33. Guevara-Carrion G., Nieto-Draghi C., Vrabec J., Hasse H., J. Phys. Chem. B, 2008, 112, 16664, doi $10.1021 /$ jp805584d

34. Schnabel T., Srivastava A., Vrabec J., Hasse H., J. Phys. Chem. B, 2007, 111, 9871, doi:10.1021/jp0720338

35. Kohns M., Horsch M., Hasse H., Fluid Phase Equilib., 2018, 458, 30, doi 10.1016/j.fluid.2017.10.034

36. Požar M., Kerasidou A., Lovrinčević B., Zoranić L., Mijaković M., Primorac T., Sokolić F., Teboul V., Perera A., J. Chem. Phys., 2016, 145, 144502, doi:10.1063/1.4964487

37. Van der Spoel D., Lindahl E., Hess B., Groenhof B., Mark A.E., Berendsen H.J.C., J. Comput. Chem., 2005, 26, 1701, doi:10.1002/jcc.20291

38. Washbrun E.W. (Ed.), International Critical Tables of Numerical Data, Physics, Chemistry and Technology, Knovel, New York, 2003.

39. Bopp P., Jancsó G., Heinzinger K., Chem. Phys. Lett., 1983, 98, 129, doi:10.1016/0009-2614(83)87112-7

40. Palinkas G., Hawlicka E., Heinzinger K., J. Phys. Chem., 1987, 91, 4334, doi:10.1021/j100300a026

41. González-Salgado D., Nezbeda I., Fluid Phase Equilib., 2006, 240, 161, doi:10.1016/j.fluid.2005.12.007

42. Pálinkás G., Bakó I., Z. Naturforsch., A: Phys. Sci., 1991, 46, 95, doi 10.1515/zna-1991-1-215.

43. Galicia-Andrés E., Dominguez H., Pusztai L., Pizio O., J. Mol. Liq., 2015, 212, 70, doi $10.1016 /$ j.molliq.2015.08.061

44. Bakó I., Megyes T., Bálint S., Grósz T., Chihaia V., Phys. Chem. Chem. Phys., 2008, 10, 5004, doi $10.1039 / \mathrm{b} 808326 \mathrm{f}$

45. Fischer N.M., van Maaren P.J., Ditz J.C., Yildrim A., van der Spoel D., J. Chem. Theory Comput., 2015, 11, 2938, doi:10.1021/acs.jctc.5b00190

46. Biscay F., Ghoufi A., Malfreyt P., J. Chem. Phys., 2011, 134, 044709, doi 10.1063/1.3544926

47. Derlacki Z.J., Easteal A.J., Edge A.V.J., Woolf L.A., Roksandic Z., J. Phys. Chem., 1985, 89, 5318, doi $10.1021 / \mathrm{j} 100270 \mathrm{a} 039$.

48. Neumann M., Mol. Phys., 1983, 50, 841, doi:10.1080/00268978300102721

49. Albright P.S., Gosting L.J., J. Am. Chem. Soc., 1946, 68, 1061, doi $10.1021 /$ ja01210a043

50. Alejandre J., Chapela G.A., Saint-Martin H., Mendoza N., Phys. Chem. Chem. Phys., 2011, 13, 19728, doi $10.1039 / \mathrm{C} 1 \mathrm{CP} 20858 \mathrm{~F}$

51. Salas F.J., Méndez-Maldonado G.A., Núñez-Rojas E., Aguilar-Pineda G.E., Domínguez H., Alejandre J., J. Chem. Theory Comput., 2015, 11, 683, doi $10.1021 / \mathrm{ct500853q}$.

52. Gofurov Sh., Ismailova O., Makhmanov U., Kokhkharov A., Int. J. Chem. Mol. Nucl. Mater. Metall. Eng., 2017, 11, 330 .

\title{
Моделювання методом молекулярної динаміки властивостей сумішей вода - метанол. Вплив силових полів
}

\author{
М. Круз Санчес ${ }^{1}$, г. Домінгес $\stackrel{2}{2}$, О. Пізіо ${ }^{1}$ \\ 1 Інститут хімії, Національний автономний університет м. Мехіко, Мехіко, Мексика \\ 2 Інститут матеріалознавства, Національний автономний університет м. Мехіко, Мехіко, Мексика
}

\begin{abstract}
Моделювання методом молекулярної динаміки в ізотермічно-ізобаричному ансамблі застосовано до дослідження мікроскопічної структури та деяких властивостей рідкої суміші вода-метанол. Моделі води TIP4P/2005 і SPC/E поєднано з моделлю об'єднананих атомів TraPPE і моделлю силових полів всіх атомів для метанолу. Основною метою даної роботи $є$ дати якісну оцінку передбаченням різних комбінацій модельних силових полів стосовно концентраційних залежностей основних властивостей системи. Зокрема, ми дослідили вплив концентрації на густину, надлишковий молярний об'єм і надлишкову ентропію, а також на поверхневий натяг і статичну діелектричну сталу. Крім цього, описано структурні властивості на мові координаційних чисел і середнього числа водневих зв'язків між молекулами компонентів суміші. Нарешті, здійснено оцінку концентраційної залежності коефіцієнтів самодифузії компонентів. Усі теоретичні передбачення перевірено по відношенню до експериментальних даних.
\end{abstract}

Ключові слова: суміші вода-метанол, властивості змішування, поверхневий натяг, моделювання методом молекулярної динаміки 